\title{
Formación en Valores Compromiso en la Universidad de la Guajira
}

\section{Values Commitment Training at the University Of Guajira}

\begin{abstract}
Annnherys Paz Marcano
PhD. Ciencias Gerenciales. Postdoctora en Gerencia de las Organizaciones. Participante del Postdoctorado de Integración y desarrollo en América Latina. Msc. Gerencia Recursos Humanos. Lcda. Administración de Empresas. Docente e investigadora de la Universidad de la Guajira-Colombia.Email:aipaz@uniguajira.edu.co; annheryspaz@hotmail.com
\end{abstract}

\section{Horacio Suarez Barros}

PhD. Ciencias Gerenciales, Msc. Gerencia Empresarial. Ingeniero Industrial. Posdoctor en Ciencias Humana. Docente e Investigadorde la Universidad de la Guajira.E-mail:hsuarez@uniguajira.edu.co; horsuarez@hotmail.com

\section{Belkis Vanegas Sprockel}

PhD. Ciencias de la Educación. Postdoctor Ciencias Humanas. Trabajadora social. Esp. Recursos Humanos. Msc. Administración de Empresas. Email: bvanegas@uniguajira.edu.co

Para citar este artículo: : Paz, A; Suarez, H \& Vanegas, B (2016) formación en valores compromiso en la universidad de la Guajira, Escenarios, Vol. 14 No 1 p,p 51-62

DOI : http:/ / dx.doi.org/10.15665/esc.v14i1.877

Recibido: febrero 5 de 2016

Aprobado: marzo 30 de 2016

\section{RESUMEN}

El presente artículo tuvo como propósito analizar la formación en valores en la Universidad de la Guajira. Metodológicamente fue un paradigma positivista, mediante un estudio de tipo analítico, descriptivo, diseño no experimental, transaccional de campo, la población fue de 3880 sujetos, muestra de 1086, con muestreo aleatorio de 39 estudiantes de octavo y noveno semestres del programa de administración de empresas del turno diurno, el instrumento un cuestionario de 20 ítems en opciones de respuestas múltiples, validado por 10 expertos y aplicado la confiabilidad por formula de Alfa Cronbach, dando 0,95. El análisis de información fue a través de estadística descriptiva. A manera de conclusión, se expresan que la formación en valores, son sistemas vivos donde la formación axiológica permite potenciar el desarrollo integral del ser humano, alineando los valores personales con los profesionales, pudiendo insertarse desde su comportamiento en el entorno laboral y crear un modelo de convivencia social por el bien común .

Palabras Clave: Formación en valores, compromiso, universidad. 


\section{ABSTRACT}

Abstract

This article was aimed to analyze values education at the University of Guajira. Methodologically was a positivist paradigm, through a study of analytical and descriptive, not experimental, transactional field design, the population was 3880 subjects sample of 1086, with a random sample of 39 students of eighth and ninth semesters of management program business day shift, the instrument a questionnaire of 20 items in multiple response options, validated by 10 experts and implemented reliability by Cronbach Alpha formula, giving 0.95. Data analysis was by descriptive statistics. In conclusion, they expressed that values education, are living systems where axiológica training allows enhance the integral development of the human being, aligning personal values with professionals, can be inserted from their behavior in the workplace and create a model of social coexistence for the common good .

Keywords: Training in values, commitment, university.

\section{INTRODUCCIÓN}

Desde siempre el hombre se ha evocado a buscar desde sus competencias medios que el generen valor agregado al quehacer diario en el campo laboral, donde sus capacidades se conviertan en una guía de vida para insertarse al trabajo, de tal manera que las nuevas tendencias en los avances del mundo globalizado, llevan consigo evolución en los factores políticos, sociales, económicos de la nación entre ellos, los de orden tecnológico, innovación y comunicación, entre otros; transformándose el uso de estos en una exigencia personal para ser de su formación profesional un reto en constante aprendizaje para alcanzar el éxito.

En este sentido, el estudio de las personas y la intervención de las mismas en el proceso de prestación de servicio en las empresas, ha demostrado que la formación profesional comprende un indicador de la excelencia de su gestión, sean de naturaleza industrial, comercial o de servicio; grandes o pequeñas, pertenezcan al sector público o privado, ya que el hombre configura el pilar fundamental en la gestión de las actividades de trabajo, por lo que el aporte de ideas en el cumplimiento de sus responsabilidades simbolizan un compromiso que las hacen competitivas en el mercado donde dispone sus bienes, productos o servicios, y con ello lograr los objetivos planificados; además de la humanización empresarial.
Por tanto, las universidades como núcleo de formación de saberes desde sus diversas disciplinas, asume un rol de gestor social, el cual ha de instruir la gestión del conocimiento con sentido humanizado representando un indicador para el desarrollo, en el cual la formación de las personas, crea un aporte para dar respuestas a las exigencias del entorno, coinvirtiéndose en campo dialectico, donde la gente enriquece el progreso de la nación.

Por ende, la formación profesional de las personas, nutre las competencias para lograr sus objetivos y ser competitivos en el mercado laboral, no solo para a los efectos de alcanzar el bienestar económico y proporcionar estabilidad sino ser profesionales integrales con disposición hacia la adaptabilidad en los retos que muestra las exigencias globales. Según (Alles, 2005) las cualidades de la fuerza de trabajo, serán el arma competitiva básica del siglo XXI y las personas especialidades la única ventaja competitiva perdurable. Por su parte, la organización internacional del trabajo (OIT, 1999) establece que la formación profesional se articula entre la educación y el sistema de a actividad económica.

Por ello, es importante resaltar, que es responsabilidad de las universidades, sin exclusión de la Universidad de La Guajira Colombia, formar personas para la vida donde instruir un aprendi-

1 Proyecto de resultado de la investigación titulada Formación en valores en la Universidad de La Guajira . Universidad de La Guajira, Riohacha, Colombia 
zaje en valores es el baluarte que enrique la sociedad, por cuanto ya hoy en día no solo se dice que un profesional integral es aquel que cumple con los niveles de formación académica, investigativa y social, sino es capaz de agregar valor desde un sentido humanizado, es decir que se preocupa por la situación de él y el de su entorno, además de mostrar en su campo de trabajo las competencias, conocimientos, habilidades adquiridas.

Según la Declaración mundial de educación superior de la (UNESCO, 1998) las universidades tienen dos responsabilidades, donde combinan el compromiso ético con el de anticipar el futuro, creando y difundiendo el conocimiento. Por otro lado, (Ibarra, 2001) la formación, es aquel proceso integral, donde las personas es capaz de mejorar y desempeñar un conocimiento (saberes), habilidades (saber hacer), actitudes y valores (saber ser).

De allí la importancia de la universidad de La Guajira de fomentar la formación en valores, pues aun cuando nacen del cimiento familiar también se enriquecen de la interacción en la sociedad, siendo la guía que dinamiza la intervención de la relaciones interpersonales en la sociedad. Refiere (Bello, 2008) que los valores, constituyen entidades cognoscitivas, emotivas, conductuales capaces de constituir en el ser humano una actitud hacia la vida, conformados por conocimientos, creencias, constataciones, vivencias personales, siendo un alto poder motivador, ejercidos en la acción del día a día.

Por consiguiente, cabe destacar que la universidad de La Guajira forma profesionales del hoy y para un mejor mañana, mediante el fomento de valores, que se muestra desde el instruir del aprendizaje del docente en un aula de clase hasta la convocatoria de otras técnicas como: participación en cursos, talleres que le faciliten promoverlos, para alcanzar un compromiso por una sociedad sostenible. Según (Castillo, 2006) la formación comprende una fase de la capacitación en los cuales se orienta la creación de valores y actitudes que le permiten a la persona desenvolverse tanto dentro como fuera del ambiente de trabajo.
Por esta razón, la universidad ha de conjugar sus actividades de academia, investigación y extensión en acciones que fomentan la actividad del docente en comportamientos éticos en las personas, para formar hombres comprometidos, con respeto, honestidad, responsabilidad, sentido de participación, es decir que los valores sean el cimiento de su actuación personal y profesional, y consecuentemente puedan ser verdaderos líderes éticos, forjando las bases para la comprensión de las actitudes y motivación, además de lograr influir en las percepciones del individuo con nociones de lo que debe y no debe hacer, permitiendo la interacción con la participación del equipo de trabajo.

Según la Declaración de la (UNESCO, 1998) sobre educación superior analizada en el artículo 2, la definición del rol ético de las universidades y la responsabilidad de su función anticipatoria, que las instituciones de educación superior, su personal y estudiantes deberán preservar y desarrollar sus funciones mediante el ejercicio en sus actividades del máximo rigor ético e intelectual; así como utilizar su capacidad intelectual y prestigio moral para defender y difundir activamente valores universalmente aceptados, tales como: paz, justicia, libertad, igualdad y solidaridad

Dentro de este contexto, también plantea (Morín, 2005), que la ética reconoce la triada individuo-sociedad-especie, para asumir la misión antropológica del milenio: de trabajar para la humanización del planeta, obedecer a la vida, guiar la vida, lograr la unidad planetaria en la diversidad, desarrollar la ética de la solidaridad, desarrollar la ética de la comprensión y enseñar la ética del género humano. En virtud, de los aspectos expuestos, el presente artículo, analiza formación en valores compromiso en la Universidad de La Guajira

\section{Formación en Valores en La Universidad de la Guajira.}

La palabra valor en el castellano y en otras lenguas románticas, tienen tres excepciones diferentes pero complementarias, las cuales (García \& Dolan,2003), exponen en tres dimensiones, la 
primera ético estratégico, la cual se refiere a los valores como aprendizaje estratégicos relativamente estable en el tiempo, de que una forma es mejor que otra; la segunda es la económica que es el alcance o significancia de una cosa, por ultima la psicológica, está referida a la valentía o a la ausencia de miedo y temores para alcanzar las metas.

Seguidamente, (Cortina, 2005), refiere que los valores se presentan tanto positivos como negativos, y con dinamismo, pues bien se considera que desde el mismo momento que percibimos un valor captamos al mismo tiempo si es positivo o negativo, ya que en el primer caso agrada, por ello atrae, en el segundo desagrada y eso repele, por tanto la autora considera, que los valores positivos incitan a las personas a alcanzarlos, mientras los negativos a radicarlos.

En tal sentido, las personas, para llevar a práctica valores dirige sus acciones hacia la transparencia y objetividad de todo cuanto hace en sus compromisos y responsabilidades adquiridas en el quehacer diario, no solo desde las esferas laborales sino como persona integral al servicio de una sociedad. Según (Schwartz, 1999), los valores son conceptos o creencias correspondientes a intenciones o comportamientos que, trascendiendo las situaciones concretas, sirviendo de guía para la selección o evaluación de comportamientos y acontecimientos priorizados en función de su importancia relativa.

Es incluso importante acotar, que la relación docente -alumno siembra su base en valores, generando un campo simbiótico entre alumno y docente y viceversa, para crear un ambiente de compromiso compartido hacia el cumplimiento de las actividades planificadas que lo conllevan obtener los objetivos y lograr el desarrollo integral como persona y profesional desde un ambiente de convivencia y humanizado. Por esta razón, los valores han demostrado ser un elemento estratégico para el progreso y desarrollo de las personas, otorgando relevancia al valor ético como fundamento ante el compromiso de las personas con el entorno.

Por lo tanto, es importante al igual que las uni- versidades, sin exclusión de La Guajira promuevan valores fundamentales, pues estos les permiten mantenerse con principios orientadores que definen en la declaración de los derechos y deberes de quienes participan en la comunidad institucional, como una de las acciones estratégicas que sustenta su responsabilidad ante el compromiso genuino de las personas con los objetivos, potenciando la motivación para el ejercicio eficiente de las actividades asignadas.

Si bien es cierto, los valores constituye el patrón de comportamiento del hombre en su entorno, por lo que su actuar se ve condicionado por la practicidad axiológica, representando la esencia de su ser para lograr su desarrollo en la formación de la vida personal y profesional. De ahí la necesidad del individuo en plantearse valores en su realidad, como creencia y principio humanístico en su contacto con la sociedad.

En cuanto a los criterios planteados, por (Negrón, Leiva \&Nava, 2010) la importancia de los valores radica en la razón de ser del hombre y es el valor moral el que más influye en la forja de la personalidad del individuo dentro de su comportamiento responsable en la sociedad. Es de gran interés tomar en cuenta que, los valores se consiguen como pauta y guía de la conducta del hombre en su relación con su entorno familiar, social y con el medio ambiente, basándose en principios y reglas de conducta ética.

Por otro lado, (Celis, 2015) los valores son palabras con fuerza especial que dan sentido y encauzan los esfuerzos humanos, siendo una convicción o creencia estable en el tiempo de un determinado modo de conducta o finalidad existencial, por tanto son absolutos y universales, los cuales se adoptan en la educación, en el hogar, en la escuela, en la socialización, estos pueden ser individuales, morales, relaciones sociales, relaciones interpersonales.

En este sentido, en los valores individuales, se identifica la fortaleza, laboriosidad, perseverancia y humildad; en relación a los tipos morales, se muestra la justicia, respeto, responsabilidad y honestidad; los valores de relaciones sociales, está la amistad, agradecimiento, bondad y gene- 
rosidad; mientras los valores de relaciones interpersonales, comprende la paz, tolerancia, solidaridad, libertad y lealtad.

Para (Ortega \& Minués, 2003), los valores permiten orientar el comportamiento en función de realzarnos como personas; además, afirman que los valores no se imponen, solo se proponen y se ejemplifican vivencialmente en el contacto con la sociedad, siendo ejemplo de ello :valores del fundador, valores de dirección, valores de los empleados, valores sociales. Asimismo, quizás ninguna persona será reconocida por el sentido único y personal que descubra en sus acciones, sino por su integración a los valores sentidos socialmente compartidos, pues estos representan un padrón de la vida heredable, haciendo de cada ser humano un actuar genuino por naturaleza ante la sociedad y la cultura.

Entonces, es tarea importante en la universidad de La Guajira la formación de personas en valores, ya que esta trabaja por una educación de academia con proyección social, es decir para una sociedad sostenible donde prevalezca la convivencia, paz, solidaridad, tolerancia, honestidad, transparencia, respeto a la diversidad, capacidad de diálogo, resolución de conflictos, participación social, desarrollo de la autonomía y auto afirmación individual y colectiva.

La formación en valores debe ocuparse del clima organizacional, así como del desarrollo de principios de respeto, equidad y solidaridad, la realización personal, la justicia social, el trabajo en equipo, entre otros. (Álvarez, et al 2010, citado por Paz, et al 2013) una faceta de la responsabilidad, la ética y la moral universitaria tiene significado propio, ya que sintetiza todos los valores y atributos propios de su responsabilidad.

Refiere, (Urdaneta, 2010, citado por Paz, et al 2015) que los valores terminales o finales son estados deseables, metas que una persona desea alcanzar durante su vida, siendo también conocidos como valores de meta, entre ellos está: la realización personal, amistad, generosidad, justicia social y solidaridad entre otros. Agrega, el autor que los valores instrumentales representan modos de comportamiento, expresando para al- canzar los valores terminales, entre ellos se conocen: la honestidad, responsabilidad, respeto, inteligencia, flexibilidad y el trabajo en equipo.

Por su parte, (Celis, 2015) establece que otra tipología de valores son los terminales e instrumentales, los primeros se refieren a los estados finales de la existencia, se trata de las metas que una persona quisiera conseguir a lo largo de su vida; mientras los segundos señalados como instrumentales son los modos preferibles de comportarse o los medios para conseguir los valores terminales.

Asimismo (García \& Dolan, 2003), existen dos tipos de valores, finales e instrumentales, los primeros tratan de la existencia del logro de los objetivos que los individuos desean alcanzar, estando asociados a la visión hacia donde van las personas y la misión (razón de ser o para que); además son esenciales para dar sentido y coherencia de determinar el esfuerzo hacia dónde va y como se quiere llegar a ser. De manera se expresa que los valores finales, se expresan de manera personal y ético social.

- Valores Personales: comprenden las aspiraciones del individuo para sí mismo, entre ellos se pueden mencionar vivir, felicidad, salud, salvación, familia, éxito, realización personal, tener prestigio, demostrar estatus, bienestar material, sabiduría, amistad, trabajo, ser respetado, demostrar valía, amor entre otros. En este sentido, se explica que en su conjunto representan el proyecto de vida de las personas expresándose a través de las interrelaciones e intervenciones en la sociedad.

- Valores Éticos Sociales: son propósitos que contribuyen a la formación del tejido social, siendo indispensables para armonizar la convivencia colectiva, ya que suelen practicarse ante el relacionar con los demás, por lo que entre ellos pueden mencionarse: el respeto, medio ambiente o ecología, respeto hacia los derechos humanos, la paz, supervivencia, justicia social entre otros.

En referencia a los segundo, el cual se refiere a los valores instrumentales estos son el modo de conducta para alcanzar las finalidades o valores 
existenciales, donde su practicidad se convierten en modos operativos para obtener los valores. De allí, que sean modos de actuar para alcanzar propósitos personales, por ello se asocian a la forma de pensar y establecer las cosas con la que la organización pretende afrontar las demandas del entorno e integra sus tensiones internas hacia la excelencia del negocio desde una filosofía organizacional compartida.

- Valores Éticos Morales: Se refiere a los modos de conducta necesaria para alcanzar los valores finales y no son necesariamente en sí mismo fines existencias, de hecho, la palabra moral proviene del latín mores, que significa costumbre. Los valores morales son de tipo de valor instrumental que tienen dos características peculiares, se ponen en práctica en relación con las demás personas; en las personas mentalmente equilibradas cuando se incorporan pero no se traducen en conductas consecuentes y en cuanto tienden a generar sentimientos de culpabilidad, o al menos malestar consigo mismo, entre este tipo de valores se atribuye la honestidad, educación con los demás, sinceridad, responsabilidad, lealtad, solidaridad entre otros.

Es por ello que, educar en valores supone transmitir conceptos que tiene la capacidad de condesar en su formulación motivaciones, intensiones, propósitos y adhesiones. Pues bien, el valor es mucho más que un concepto del intelecto, este tiene la capacidad de afectar al ser humano como un ser integral, es decir como un intelectual y sensible. Entonces, educar en valores, es en tanto un aprendizaje para la vida en el rigor lógico que debe conducir elecciones como una enseñanza en el sentimiento y las emociones, entonces ayudan a identificar las situaciones de la práctica ética en la vida misma y su conocimiento moral, .ayudando a operativizar la actuación ante la sociedad, desde la configuración de su proyecto de vida.

- Valores de Competencia: Estos son más individuales, aunque también están socialmente condicionados y no están directamente relacionados con la moralidad ni la culpabilidad. Es por ello que los autores sostienen: "Nadie se siente culpable, ni tan solo, ni cómodo, por no asumir un valor que sea compartido por un grupo de personas del cual se desea formar parte". Es importante resaltar que, los valores por competencia representan el valor interno que una persona posee, traducidos en conducta, lo que permite trasmitir sus experiencias con el resto de los trabajadores.

- Ahora bien, al hablarse sobre la formación de talento humano en valores, se refiere al desarrollo del potencial humano a su máximo nivel al alinear valores personales y corporativos e incorporarlo como ciudadano corporativo para crear riqueza económica y social en un marco de equilibrio y estabilidad para la excelencia organizacional, aunado a la humanización del ambiente laboral. Mencionan (Urdaneta \& Alfonzo, 2010) que las organizaciones que aspiren al éxito deberán considerar la importancia de formar y desarrollar individuos quienes valoren realmente lo que hacen, y como lo hacen; de esta manera las personas se sentirán útiles y apreciaran la organización considerándola como suya.

\section{Metodología.}

El presente artículo, se desarrolló mediante la práctica de actividades investigativas para conocer sobre información asociada al tema objeto de estudio en este caso la formación en valores en la universidad de La Guajira, a través de criterios de reflexión analítica y critica para cumplir con el objetivo planteado. Según (Hernández, Fernández, Baptista, 2014) la recolección de datos, implica elaborar un plan detallado de procedimientos que conducen y reúnen datos con un propósito.

En este particular se presenta con un enfoque epistemológico cuantitativo, positivista, interpretando hechos de la realidad, a través de los datos a suministrados por las unidades informantes. En cuanto al paradigma, señala Behar (2009) que implica el conjunto de ideas preconcebidas, tendencias de pensamiento y/o patrones de investigación compartidos. En este sentido el estudio suscribe en el paradigma cuantitativo de origen positivista, según los criterios de Rojas (2014) se sustentan como la doctrina de la ciencia, bajo la teoría del saber, en el cual no 
se admite otra realidad que no sean los hechos y la relación entre ellos. Según Behar (2009) el paradigma cuantitativo, recoge información empírica (de cosas o aspectos que se pueden contar, pesar o medir) y que por su naturaleza siempre arroja números como resultado.

Sobre la base del objetivo planteado, se orienta como descriptiva y alude a la orientación de la acepción de información compilada asociada a la variable objeto de estudio, desde la opinión objetiva de las unidades informantes.

. Para (Hurtado, 2008), la investigación analítica reinterpreta criterios, dependientes de los objetivos en estudio. Por su parte, (Chávez, 2007), con respecto a los estudios descriptivos señala que estos se orientan a recolectar información relacionada con el estado real de las personas, objetos, situaciones o fenómenos, tal cual como se presentaron en el momento de recolección describe que se mide sin realizar inferencias ni verificar hipótesis, este tipo de investigación permite llevar a cabo una descripción, análisis sobre los datos obtenidos referente a la variable de estudio, partiendo de características particulares que facilitan su gestión en el escenario de investigación.

El diseño del estudio se suscribe, como no experimental, transaccional de campo, donde no se manipula la información sino se obtuvo de fuentes reales en el tiempo planificado por los investigadores. Según (Hernández, et al, 2014) el diseño no experimental está destinado a observar los fenómenos tal y como se dan en su contexto natural, para después interpretarlo. Por otra parte, (Bavaresco, 2006) establece que la investigación de campo, se realiza en el propio sitio donde se encuentra el objeto de estudio, permitiendo un conocimiento a fondo del problema investigado, generando seguridad en los datos recolectados de la realidad.
En cuanto a la búsqueda de información sobre los aspectos de la variable formación en valores, se limitó a la opinión de los estudiantes de la Facultad de Ciencias Económicas y Administrativas de la Universidad de la Guajira sede central Riohacha Departamento de la Guajira. (Hernández, et al, 2014) la población es el conjunto de todos los casos que concuerdan con determinadas especificaciones, partiendo de características de contenido, de lugar y el tiempo.

En la población se consideró a los estudiantes de la Facultad de Ciencias Económicas y Administrativa de la Universidad de la Guajira, sede central, el cual corresponde a 3880 sujetos. En relación a la muestra, fue de 1086 sujetos del programa de Administración de Empresas, en el cual se aplicó un muestreo aleatorio en los estudiantes de 8 vo. y 9vo. Semestre del mencionado programa, en el turno diurno estando representado a los efectos de investigación por 39 sujetos.

En relación a compilación de información acerca de la variable formación en valores, se aplicó como técnica la encuesta e instrumento un cuestionario de 20 ítems, con opciones de respuestas múltiples de siempre, casi siempre, a veces, y nunca. Cabe señalar, que el instrumento fue validado por 10 expertos y calculado la confiabilidad por la fórmula de Alfa Cronbach, reflejando un resultado de 0, 95 de confiabilidad; asimismo los datos obtenidos se interpretaron con la estadística descriptiva ilustrando los datos en cuadros, siendo presentados en frecuencias absolutas y relativas, posteriormente una vez obtenidos los resultados para su posterior análisis, se construyó un baremo de interpretación con la finalidad de contrastar los datos, y luego interpretar el comportamiento de las categorías que coadyuvaron a demostrar el logro del objetivo de estudio, tal como se ilustra en el cuadro 2.

Cuadro 1: Distribución Poblacional

\begin{tabular}{cc}
\hline \multicolumn{1}{c}{ Facultad } & Número de estudiantes \\
\hline Ciencias Económicas y Administrativas & 3880 \\
\hline
\end{tabular}

Fuente: Dirección admisiones y registro de la Universidad de La Guajira (2016) 
Cuadro 2: Baremo ponderado para la interpretación de Resultados

\begin{tabular}{lc}
\hline Categoría & Rango \\
\hline Muy baja & $1,00 \geq \mathrm{x}<1,80$ \\
Baja & $1,80 \geq \mathrm{x}<2,60$ \\
Moderada & $2,60 \geq \mathrm{x}<3,40$ \\
Alta & $3,40 \geq \mathrm{x}<4,20$ \\
Muy alta & $4,20 \geq \mathrm{x} \leq 5,00$ \\
\hline
\end{tabular}

Fuente: Pallella \& Martins (2003).

\section{Resultados}

A continuación se presenta el análisis de las respuestas obtenidas por la muestra objeto de estudio; producto de la aplicación del cuestionario como instrumento compilador de datos acerca de la variable formación en valores en la univer- sidad de la Guajira, se llevó a cabo un análisis estadístico, basado en el ordenamiento descriptivo de frecuencias absolutas, proporciones y porcentajes por categoría, de los indicadores y dimensiones de las mismas.

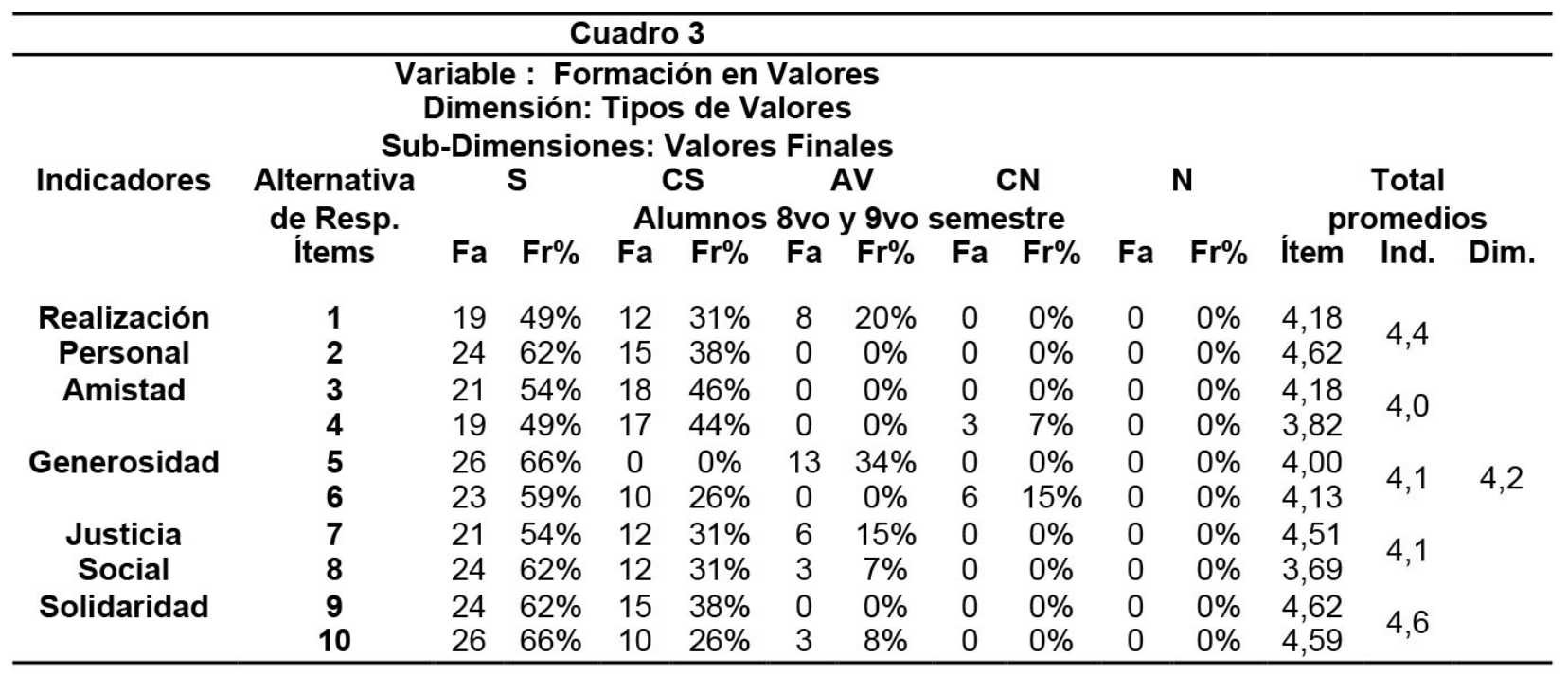

Fuente: Paz, Suarez \& Vanegas (2016)

Con respecto a la información expresada en el cuadro 3 y grafico 1, se muestran los resultados derivados de la opinión emitida por los encuestados los cuales son alumnos de octavo y noveno semestre de facultad de ciencias económicas y administrativas, del programa de administración en la universidad de La Guajira, obteniéndose en el indicador realización personal, para el ítems 1 que el $49 \%$ expreso que siempre los docentes en sus estrategias de aprendizaje promueven los valores, un $31 \%$ destaco casi siempre y un $20 \%$ expreso a veces se cumplen las mencio- nadas estrategias. En el ítems 2, un 62\% asevero que siempre los docentes entre sus planes de aprendizaje insertan los valores como parte de su interacción de formación en el aula de clase, no obstante un $38 \%$ señalo hacerlo casi siempre.

Por otra parte, en el indicador amistad el ítems 3, un 54\% manifestó que siempre se mantiene un ambiente armónico en los espacios de clase y un $46 \%$ casi siempre; por consiguiente ítems 4 un 49\% planteo que siempre se promueve la unión entre los compañeros de clase, un $44 \%$ que casi 


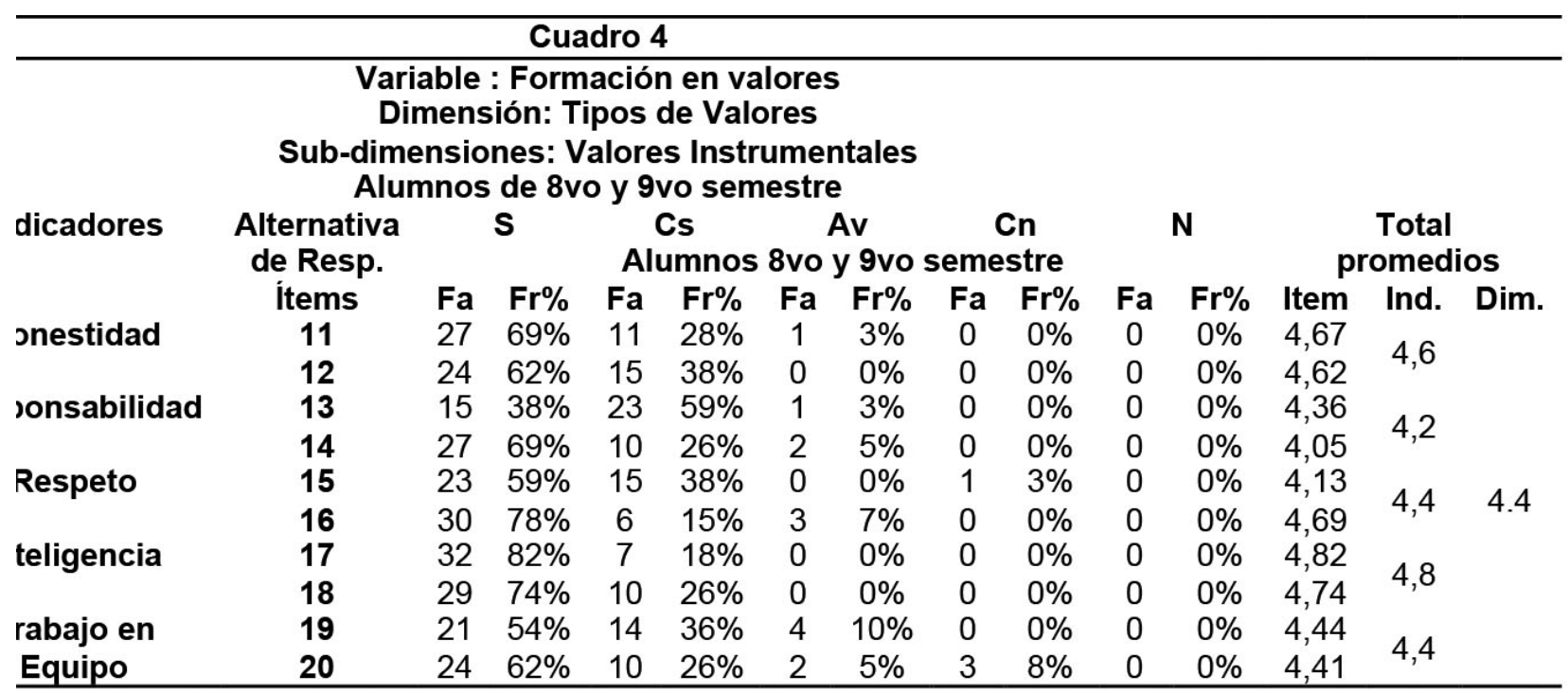

ruente: raz, suarez $\&$ vanegas ( $\angle$ U 10)

siempre y un $7 \%$ casi nunca se promueve tal situación. En el indicador generosidad, el ítem 5 un $66 \%$ de los encuestados plantearon que siempre muestran preocupación cuando algún compañero expresa alguna dificultad familiar, y un $34 \%$ casi siempre lo hacen. Seguidamente, en el mismo indicador el ítems 6, un 59\% ofrece siempre apoyo para solventar situaciones que afectan a sus compañeros, un $26 \%$ casi siempre y 15 $\%$ casi nunca realiza tal apoyo.

En relación al indicador justicia social, el ítems 7 el $54 \%$ de los encuestados planteo que siempre muestra respeto entre sus compañeros aunque no comparta su idea, un $31 \%$ casi siempre y un $15 \%$ a veces; por consiguiente en el ítems 8 un $62 \%$ indico que siempre emite su opinión ante una situación determinada en los espacios de clase aunque no sea escuchada entre sus compañeros, el $31 \%$ casi siempre y $7 \%$ a veces.

En cuanto al indicador solidaridad, el ítems 9 arrojo que un $62 \%$ admitió que siempre ejerce apoyo entre los compañeros cuando estos presentan ausencias de clases y $38 \%$ opino hacerlo casi siempre; ítems 10 cuando no entiende alguna actividad asignada por el docente busca orientación entre los compañeros, un $66 \%$ manifestó hacerlo siempre, $26 \%$ casi siempre y $7 \%$ a veces recurre a la mencionada orientación.

En correspondencia a los promedios de los re- sultados obtenidos de la opinión de los encuestados, para los valores instrumentales en la formación del talento humano en la universidad de La Guajira el valor promedio más alto fue en el indicador realización personal y justicia social, con un 4.62 siendo según la categoría del baremo de interpretación como muy alta presencia de la formación en valores de los alumnos, mientras la dimensión se ubicó en 4.2 con categoría alta.

Grafico 2. Resultados promedios de los Valores Instrumentales

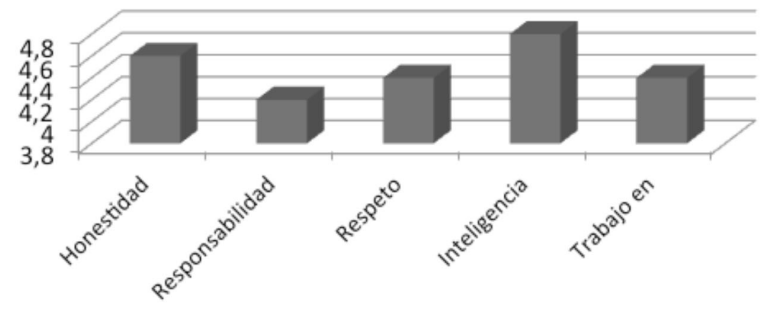

Fuente: Paz, Suarez \& Vanegas (2016)

De acuerdo a los datos del cuadro 4 y grafico 2, en el indicador honestidad, el ítems 11 un 69\% menciono que siempre actúa ante una situación como piensa, un $28 \%$ casi siempre y un $3 \%$ a veces; de acuerdo al ítems 12 un 62\% expreso que siempre los docentes promueve la transparencia al momento de asignar un trabajo, un $38 \%$ casi siempre. Por otra parte, el indicador responsabi- 
lidad, el ítems 13 un 59\% admitió que casi siempre asume las consecuencias de los actos de su desempeño académico, un 38\% siempre y 3\% a veces. En lo referente al ítems 14, un 69\% refirió que siempre cumplen con las actividades asignadas por el docente, un $26 \%$ casi siempre y un $5 \%$ a veces realizan el cumplimiento señalado.

Con respecto al indicador respeto, en el ítems 15 el $59 \%$ de los encuestados expreso que siempre permite que otros lo oriente en las actividades asignadas, un $38 \%$ casi siempre y un $3 \%$ casi nunca. Para, el ítem 16 un $78 \%$ de los alumnos encuestados señalaron que los docentes promueven la convivencia durante sus actividades académicas, un $15 \%$ casi siempre y $3 \%$ a veces. Los resultados del indicador inteligencia, en el ítems 17, que un $82 \%$ de la muestra encuestada refirió que siempre el docente promueve el uso de la creatividad mediante el aporte de ideas en la solución de casos particulares, un 18\% casi siempre. El ítems 18, el 74\% admitió que los docentes ofrecen estrategias que le aportan la generación de ideas en temas novedosos, y $26 \%$ señalo que casi siempre.

Por otra parte el indicador trabajo en equipo, se evidencio que en el ítems 19 el 54\% de los encuestados admitió que siempre los docentes promueven el intercambio de ideas entre compañero para un mejor desempeño académico, un 36\% casi siempre y $4 \%$ a veces ocurre el mencionado intercambio. En el ítems 20, un $62 \%$ señalo que siempre los docentes hacen intercambio entre los equipos de trabajo, para efectuar actividades prácticas que conlleve a solventar una situación determinada, el $26 \%$ casi siempre, $5 \%$ a veces y $8 \%$ casi nunca.

Para finalizar el análisis del comportamiento de la variable acerca de la sub dimensión valores instrumentales, se puede apreciar en la tabla 2 que el indicador de mayor valor promedio fue de 4.8 con inteligencia, siendo según la interpretación del baremo se ubicó en muy alta presencia, asimismo la dimensión con 4.4 siendo al igual muy alta presencia de los valores instrumentales en los alumnos del programa de administración de la universidad de La Guajira.

\section{Discusión de los Resultados}

Partiendo de los resultados derivados de la opinión de los encuestados se presenta a continuación la contrastación documental según los datos obtenidos. En este sentido, se hace referencia a la consulta de las fuentes teóricas desarrolladas, en el desarrollo investigativo.

Se evidencia la presencia en la formación de las personas en la universidad de La Guajira, de valores terminales lo que hace suponer un profesional integral capaz de mostrar sus competencias y transmisión de valores en el entorno, coincidiendo con la teoría de (Urdaneta, 2010) el cual plantea que los valores terminales o finales comprenden los estados deseables, metas que una persona quiere alcanzar durante su vida, siendo también denominados valores de meta, algunos de ellos suele atribuirse a la realización personal, amistad, generosidad, justicia social y solidaridad entre otros.

Asimismo, obtuvo la presencia en los encuestados de los valores instrumentales, coincidiendo con la teoría de (Urdaneta, 2010) donde los valores instrumentales representan modos de comportamiento, expresando para alcanzar los valores terminales, entre estos pueden plantearse la honestidad, responsabilidad, respeto, inteligencia, flexibilidad y el trabajo en equipo. Por otro lado, para ambos tipos de valores, se presentan aspectos similares a los enunciados por(García \& Dolan,2003), quienes explicando que existen dos tipos de valores, finales e instrumentales, los primeros tratan de la existencia del logro de los objetivos que los individuos desean alcanzar, estando asociados a la visión hacia donde van las personas y la misión (razón de ser o para que); además son esenciales para dar sentido y coherencia de determinar el esfuerzo hacia dónde va y como se quiere llegar a ser. De manera se expresa que los valores finales, se expresan de manera personal y ético social.

Agregando, también lo expuesto por Celis, 2015) establece que otra tipología de valores son los terminales e instrumentales, los primeros se refieren a los estados finales de la existencia, se trata de las metas que una persona quisiera 
conseguir a lo largo de su vida; mientras los segundos señalados como instrumentales son los modos preferibles de comportarse o los medios para conseguir los valores terminales

\section{Conclusiones}

Los valores son creencias, principios fundamentados en el hombre para su actuar social, siendo su patrón de comportamiento ante la acción de seleccionar una situación determinada sea esta positiva o negativa, por lo tanto viene hacer su modelo axiológico que lo conlleva a la motivación y la satisfacción a plenitud, proporcionando una pauta que guía la formulación de metas o propósitos personales o colectivos.

Por otro lado, la formación en valores en los estudiantes en los espacios académicos en la universidad de La Guajira, fomenta un sentido de humanización que proyecta desde su lema de academia con proyección social, siendo garante de calidad de vida en la sociedad, en armonía con un ambiente de convivencia donde se desenvuelve, permitiendo instruir profesionales que le encuentren significado a lo que se hace y un mayor compromiso hacia las labores que desempeña no solo como hombre sociable sino profesional que crea desde su actuación una convivencia colectiva.

Entonces, al hablar de valores en la formación universitaria, conlleva a un sentido de humanización mediante un modelaje de patrones de conducta específicos que pueden reconocerse, transmitirse y apropiarse de la actuación en el entorno; en otras palabras, los valores como principio comportamental de hombre y mujeres profesionales en la sociedad facilita desarrollar o modificar actuaciones, alcanzando el cambio de actitudes negativas a actitudes favorables, tomando conciencia y sensibilidad en el entorno; además, de establecer ideas y concepción para pensar en mejorar su relación consigo mismo y los demás, a objeto de fomentar el bienestar por el bien común.

Por consiguiente, la universidad de La Guajira instruye la formación del ser humano para la vida infundiendo la transmisión de valores que contribuyen a fortalecer su formación integral, es decir que ofrece desde su aprendizaje fuentes axiológicas instrumentales como son realización personal, amistad, generosidad, justicia social y solidaridad. Asimismo, valores finales como son honestidad, responsabilidad, respeto, inteligencia y trabajo en equipo donde de manera conjunta lo convierte en una persona genuina en su comportamiento en la sociedad.

\section{Referencias}

Alles, M (2005) Dirección estrategia del recurso humano. Gestión por competencias. Argentina .Editorial Granica

Bavaresco, A. (2006). Proceso metodológico en la investigación. Venezuela. Maracaibo. Editorial de la Universidad del Zulia Maracaibo - Venezuela. Quinta edición

Behar, D (2009). Metodología de la Investigación. México. Editorial Shalom

Bello, J. (2008). Valores para construir una Ética. Taller para la edificación de un mundo posible. Caracas. Venezuela. Liven Editores, C.A. Primera Edición.

Castillo, J (2006) Administración de Personal. Colombia. Editorial Presencia, Ltda.

Chávez N, (2007). Introducción a la investigación educativa. Venezuela. Maracaibo. Editorial ARS. Tercera edición.

Celis, M (2015) La conducta en las organizaciones. Bases para su estudio con una perspectiva sistemática - social. Venezuela Editorial Universidad de Carabobo. Primera Edición

Cortina, A (2005). El mundo de los valores. Ética mínima y Educación. Editorial Buho. Colombia.

García, S y Dolan, S (2003). La dirección por valores. El cambio más allá de los objetivos. España. Editorial McGraw-Hill.

Hernández, R; Fernández, C y Baptista; M (2014) Metodología de la Investigación. México .Editorial Mc. Graw Hill Interamericana Editores, S.A. Sexta Edición 
Hurtado, J (2008) Metodología de la Investigación. Venezuela. Ediciones de Sypal- Quirón. Segunda edición.

Ibarra, A (2001) Formación de recursos humanos y competitividad laboral. VII congreso Latinoamericano de Innovación educativa . Conocer México. www.oei.es/etp/formacion_recursos_ humanos_competencia_laboral_ibarra.pdf

Morin, E. (2005), Los siete saberes necesarios para la educación del futuro. Paris. UNESCO

Negrón M., Leiva M., y Nava F. (2010) Modelo de gerencia sustentado en valores Revista Científica Electrónica Ciencias Gerenciales / Scientific e-journal of Management Science PPX 200502ZU1950 / ISSN 1856-1810 / By Fundación Unamuno / Venezuela. En Línea http:/ / www. revistanegotium.org.ve/pdf/17/Art5.pdf

Organización internacional del trabajo (OIT) (1999) El enfoque de competencias laboral. Manuel de formación OIT. Agencia Española de cooperación internacional. Cinterfor, Montevideo

Ortega y Minués (2003). Los valores en la Educación. España. Editorial Ariel S.A.

Paz, A; Morillo E y Celedón, A (2015). Apertura de la Comunicación como caracterización de los Valores Organizacionales en Universidades Públicas Venezolanas, 13 (2) , p,p 7-21

Paz, A; Nuñez, M; Salóm, J y Rosales, V (2013) Responsabilidad social: moralidad o compromiso en la formación de valores éticos en universidades. Venezuela. Revista Opción, Año 29, No. 72 (2013): 97 - 116. Universidad del Zulia

Palella, S. y Martins, F. (2003). Metodología de la Investigación cuantitativa. Caracas: Fedupel.

Ramos, M (2006) Valores y Autoestima. Conociéndose a sí mismo en un mundo con otros. Venezuela .Editorial San Pablo.

Robbins, S. (2009) Comportamiento Organizacional. México. Editorial Pearson Educación. Decima tercera edición

Rojas, B (2014) Investigación Cualitativa. Fundamentos y praxis. Venezuela. Editorial de la Universidad Pedagógica Experimental Libertador. Tercera edición

Schwartz, Sh. (1999). A theory of cultural values and some implications for work. Estados Unidos. Editorial Zamma.

Silíceo A., Casares D. y González J. (2000). Liderazgo, Valores y Cultura Organizacional. México. Editorial McGraw-Hill. Primera Edición

UNESCO (1988). Declaración Mundial sobre la Educación Superior en el Siglo XXI. Aprobada por la Conferencia Mundial sobre Educación Superior celebrada en la ciudad de Paris. Disponible en: http://www.unesco.org/education/ educprog/wche/declaration_spa.htm. Consultado el 30.06.2011.

Urdaneta L. y Alfonzo F. (2010). Mercadeo social: marco estratégico de cambio en las organizaciones. Revista CICAG, volumen 7 - edición 1 - año 2010. URBE.

Urdaneta, M (2010) Sistema de Valores objetivados en los Docentes de Educación Especial. Trabajo de grado para optar al Título de Magíster Scientiarum en Educación. Mención Planificación Educativa Universidad Del Zulia. Venezuela. 